\title{
ANÁLISE DE AMEAÇA DE DESLIZAMENTOS PELO USO DE ABORDAGEM PROBABILÍSTICA APLICADA A UM MODELO DE ESTABILIDADE DE TALUDES TRIDIMENSIONAL
}

\author{
LANDSLIDES HAZARD ANALYSIS BY USE OF APPROACH PROBABILISTIC APPLIED TO A \\ THREE-DIMENSIONAL SLOPE STABILITY MODEL
}

\section{George Fernandes AZEVEDO, Hernán Eduardo Martinez CARVAJAL, Newton Moreira de SOUZA} \author{
Email: george_engenheiro@hotmail.com; carvajal@unb.br; nmsouza@unb.br

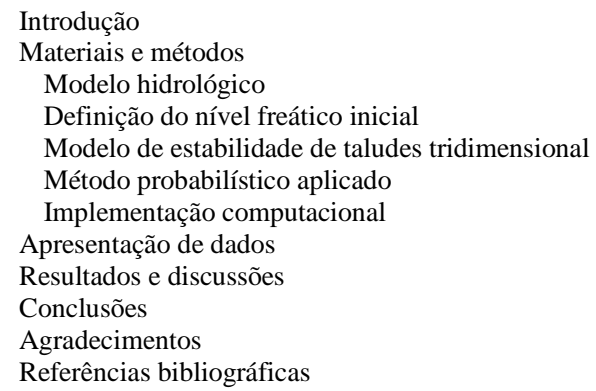

Programa de Pós-Graduação em Geotécnica, Departamento de Engenharia Civil e Ambiental, Universidade de Brasília.

RESUMO - Este artigo aplica uma formulação probabilística a um modelo de estabilidade de taludes tridimensional para definir a ameaça quantitativa associada a deslizamentos rasos em termos de probabilidade de ruptura. A análise do problema utiliza um modelo de equilíbrio limite 3D de estabilidade de taludes que considera a ação da precipitação por meio de modelos de infiltração capazes de simular o avanço da frente úmida e a variação do nível freático em função de um dado evento pluviométrico. De forma adicional, a probabilidade de ruptura foi obtida pelo uso de um método probabilístico das estimativas pontuais, que insere a variabilidade de alguns parâmetros do solo e fornece a distribuição probabilística do fator de segurança. A metodologia foi empregada para o mapeamento da ameaça de deslizamentos deflagrados por chuvas em uma região do Distrito Federal, Brasil, considerada com alto potencial de ocorrência de eventos desta natureza. Como resultado, configurações com a distribuição das probabilidades de rupturas foram alcançadas, possibilitando distinguir a condição de estabilidade das encostas envolvidas no estudo. Palavras-chave: Deslizamentos, Ameaça, Fator de segurança tridimensional, Método das estimativas pontuais, Chuva.

ABSTRACT - This paper applies a probabilistic formulation to 3D slope stability model to define the quantitative hazard associated to shallow landslides in terms of probability of failure. The problem analysis uses a 3D limit equilibrium model of slope stability considering the action of rainfall by infiltration models able to simulate advancing wetting front and the change in the groundwater table due to a given rainfall event. Additionally, the probability of failure was obtained by using a probabilistic method (point estimative method) that inserts the variability of certain parameters of the soil and provides the probability distribution of factor of safety. The methodology has been applied for mapping the landslides hazard triggered by rains in a region of the Federal District, Brazil, considered with high potential for occurrence of such events. As a result, configurations of the spatial distribution of probability of failure were achieved, making it possible to distinguish the condition of slope stability in this study.

Keywords: Landslides, Hazard, 3D Factor of safety, Point estimative method, Rain.

\section{INTRODUÇÃO}

Os movimentos de massa configuram-se como eventos de caráter potencialmente desastroso, principalmente em zonas tropicais e montanhosas, uma vez que a susceptibilidade do terreno em desencadear estas formas de processo aumenta em função das características fisiológicas do relevo e das atividades antrópicas (Schuster, 1996). A fisiologia pode ser entendida como a condição atual do relevo, a qual é determinada pelos processos morfodinâmicos atuantes na encosta e suas derivações resultantes (como os próprios deslizamentos).

A crescente e indiscriminada ocupação urbana que se processa em áreas desfavoráveis, sem o apropriado planejamento do uso do solo e com a ausência da incorporação de técnicas adequadas de estabilização, tem acarretado o desenvolvimento de acidentes vinculados a processos desta natureza, que, por diversas vezes, tomam proporções de desastres (Tominaga, 2007).

De forma geral, a maioria dos trabalhos que envolvem o estudo de movimentos de massa utiliza uma equação de taludes infinitos unidimensional apara analisar a estabilidade de taludes (Collins \& Znidarcic, 1998; Cho \& Lee, 2002; Frattini et al., 2004). Dentro do domínio da engenharia geotécnica, também é comum se realizar análises de estabilidade de taludes pelo uso de métodos de equilíbrio limite bidimen- 
sionais, de forma que o fator de segurança (FS) é avaliado utilizando-se uma representação 2D do talude (Xie et al., 2011).

No entanto, Qiu et al. (2006) destacam que nos casos onde a geometria do talude e a superfície de ruptura variam significantemente na direção lateral, ou quando as propriedades do material são altamente anisotrópicas ou, ainda, onde a encosta é carregada localmente, a situação tridimensional deve ser considerada. Apesar de variados métodos 3D terem sido propostos no meio técnico, um modelo tridimensional prático para a análise de estabilidade em termos computacionais ainda é requerido.

Nesta perspectiva, o presente trabalho se baseia na implementação computacional de um modelo para a análise de estabilidade de taludes $3 \mathrm{D}$, que atua em conjunto com um modelo hidrológico capaz de considerar as mudanças nos parâmetros geotécnicos do solo em função da precipitação atuante. $\mathrm{O}$ modelo de estabilidade é modificado para incorporar a variabilidade de alguns parâmetros que o compõe, por meio do método probabilístico das estimativas pontuais. Ao contrário da abordagem determinística, a qual resulta em um único valor de fator de segurança, a aplicação da análise probabilística permite gerar uma distribuição probabilística para o fator de segurança 3D, com a consequente possibilidade de quantificação da confiabilidade da encosta.

\section{MATERIAIS E MÉTODOS}

A metodologia proposta no presente trabalho consistiu na pesquisa e implementação computacional de modelos capazes de fornecer uma análise de estabilidade de taludes em termos tridimensionais, sob a forma de probabilidade de ruptura (PR). Os códigos computacionais foram gerados com o auxílio do software MATLAB (Matrix Laboratory).

De forma adicional, fez-se a aplicação a um caso de estudo no sentido de se averiguar o comportamento preditivo e de desempenho referente à ferramenta desenvolvida. A descrição dos modelos e procedimentos aqui empregados encontram expostos nas seções seguintes.

\section{Modelo Hidrológico}

Apesar da infinidade de modelos disponíveis para a avaliação da infiltração com base nas características do solo, Qiu et al. (2006) ressaltam que a quantificação deste processo, bem como a sua influência na estabilidade dos taludes, em termos regionais, ainda são difíceis, em função, principalmente, dos muitos parâmetros utilizados e da complexidade inerente ao mecanismo do fenômeno.

Da mesma forma que Qiu et al. (2006), partindo-se do entendimento que a infiltração das chuvas pode ser considerada aproximadamente vertical através da zona não-saturada antes de chegar ao lençol freático ou à camada impermeável, adotou-se, neste trabalho, o modelo de infiltração unidimensional proposto por Green \& Ampt (1911).

A este modelo, de uso relativamente simples, pode-se vincular cinco hipóteses principais: (1) a superfície do solo é mantida constantemente úmida pelo acúmulo de água; (2) existência de uma frente úmida acentuada; (3) a condutividade hidráulica é constante ao longo da profundidade; (4) a matriz de sucção do solo na frente úmida permanece constante; (5) o solo encontra-se totalmente saturado desde a superfície até a profundidade da frente úmida, em função da infiltração.

A camada de solo abaixo da frente úmida está em um grau de saturação inicial inferior a condição saturada. A taxa de infiltração f pela lei de Darcy é dada por:

$$
f=K_{S} \frac{\psi_{f}+Z_{f}}{Z_{f}}
$$

onde $K_{S}$ é condutividade hidráulica do solo (em $\mathrm{m} / \mathrm{h}), \psi_{f}$ corresponde a matriz de sucção na frente úmida (em $\mathrm{m}$ ) e $Z_{f}$ refere-se a profundidade da frente úmida (em $\mathrm{m})$.

Pode-se relacionar a quantidade acumulada de água infiltrada $F$ (em m) com a profundidade da frente úmida pela seguinte equação:

$$
F=Z_{f}\left(\theta_{S}-\theta_{i}\right)
$$

onde $\theta_{i}$ e $\theta_{S}$ relacionam-se ao teor de umidade volumétrico inicial e o teor de umidade volumétrico saturado do solo, respectivamente. A taxa de infiltração em qualquer tempo t é dada pela próxima expressão, a qual pode ser obtida isolando-se a variável $Z_{f}$ na Equação 2 e substituindo-a na Equação 1, conforme mostrado abaixo.

$$
f(t)=K_{S}+K_{S} \frac{\psi_{f}\left(\theta_{S}-\theta_{i}\right)}{F}
$$


A quantidade acumulada de água infiltrada em um determinado tempo $t$ pode ser escrita da seguinte forma:

$$
F(t)=t_{p}+\frac{1}{K_{S}}\left[F-F_{p}+\psi_{f}\left(\theta_{S}-\theta_{i}\right) \ln (n)\right]
$$

onde $n$ é representada pela equação abaixo:

$$
n=\frac{\psi_{f}\left(\theta_{S}-\theta_{i}\right)+F_{p}}{\psi_{f}\left(\theta_{S}-\theta_{i}\right)+F}
$$

Os termos $t_{p}$ e $F_{p}$ correspondem às expressões seguintes:

$$
\begin{gathered}
{ }_{p}{ }_{p}=\frac{F_{p}}{P} \\
F_{p}=\frac{\psi_{f} K_{S}\left(\theta_{S}-\theta_{i}\right)}{P-K_{S}}
\end{gathered}
$$

$F_{p}$ refere-se à quantidade de água que infiltra no solo antes que ocorra o acúmulo de água na superfície, enquanto $t_{p}$ é o tempo necessário para que ocorra o início da acumulação de água na superfície. O termo $P$ representa a intensidade de precipitação, em m/h. Conforme Qiu et al. (2006), observa-se que a intensidade da chuva deve ser maior que a condutividade hidráulica do solo para que as equações 3 a 7 sejam satisfeitas. A intensidade de precipitação torna-se igual à taxa atual de infiltração, com toda chuva infiltrando no solo sem runoff, no caso em que $P \leq K_{s}$ (taxa de chuva inferior à taxa potencial de infiltração) ou $t$ $\leq t_{p}$ (quando não ocorre o acúmulo de água na superfície sob a forma de poças), conforme expresso nas equações a seguir:

$$
\begin{aligned}
& f(t)=P \\
& F=P . t
\end{aligned}
$$

Para o cálculo da profundidade de infiltração em um determinado tempo, pode-se utilizar um processo iterativo referente à Equação 4 ou aplicar uma solução aproximada desta equação proposta por Serrano (2003). Esta última abordagem fornece resultados muito próximos da solução exata nos casos em que a intensidade de chuva não é tão elevada quando comparada com a condutividade hidráulica saturada do solo. As expressões abaixo representam esta abordagem:

$$
\begin{gathered}
F(t) \approx F_{0}(t)+\psi_{f}\left(\theta_{S}-\theta_{i}\right) \ln \left(m_{1}(t)\right)\left(1+\frac{m_{2}(t)}{\left[1-m_{2}(t)\right]\left\{1+m_{2}(t) \ln \left[m_{1}(t)\right]\right\}}\right) \\
F_{0}=K_{S}\left(t-t_{p}\right)+F_{p} \\
m_{1}(t)=\frac{F_{0}(t)+\psi_{f}\left(\theta_{S}-\theta_{i}\right)}{F_{p}+\psi_{f}\left(\theta_{S}-\theta_{i}\right)} \\
m_{2}(t)=\frac{\psi_{f}\left(\theta_{S}-\theta_{i}\right)}{F_{0}(t)+\psi_{f}\left(\theta_{S}-\theta_{i}\right)}
\end{gathered}
$$

Observa-se que as equações 4 e 10 possuem um elevado nível de concordância quando a seguinte condição é verificada:

$$
2 m_{2}(t)<1
$$

Utiliza-se, então, a Equação 10 ou a Equação 4 para a determinação da quantidade acumulada de água infiltrada em um dado tempo, caso a condição estabelecida na Equação 14 seja satisfeita ou não, respectivamente.

\section{Definição do Nível Freático Inicial}

Para a estimativa da camada referente ao nível freático inicial, utilizou-se a formulação hidrológica do modelo SLIDE (SLopeInfiltration-Distributed Equilibrium) proposto por Liao et al. (2010) com base no trabalho de

Montrasio \& Valentino (2008). A representação esquemática deste modelo se encontra na figura 1.

Na figura 1, $\mathrm{H}$ representa a espessura do solo acima da camada impermeável (embasamento rochoso ou solo rígido em profundidade), referente aquela que irá deslizar. O modelo SLIDE corresponde à base de um sistema de alerta para previsão de escorregamentos rasos deflagrados pela ação de precipitações, aplicado na Ilha de Java, situada na Indonésia. Fundamenta-se no método de equilíbrio limite referente a taludes infinitos, considerando hipóteses simplificadas para o fluxo subsuperficial e definindo uma correlação direta entre o fator de segurança das encostas e a quantidade de precipitação. 


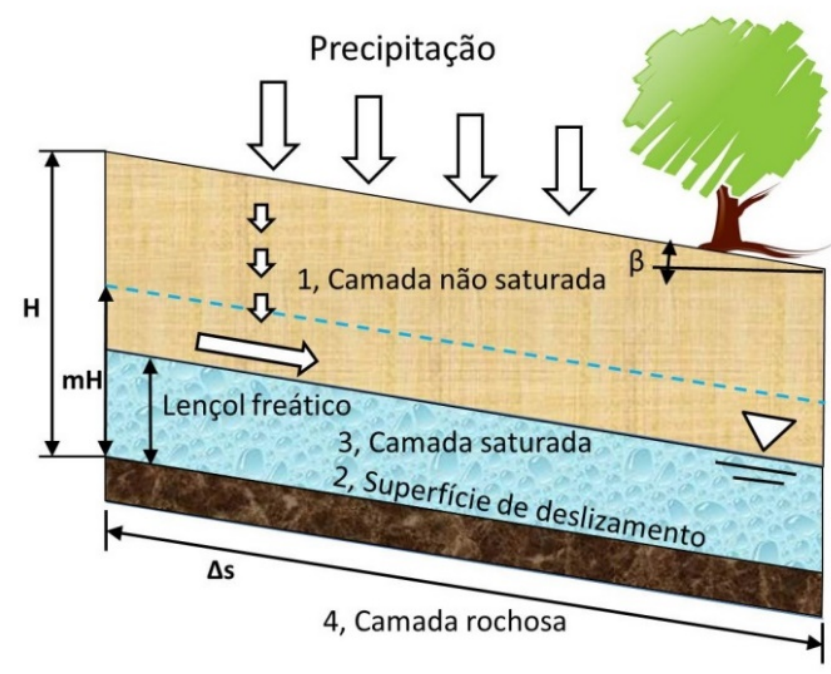

Figura 1 - Esquema representativo para o modelo SLIDE (modificado de Liao et al., 2010).

Conforme Montrasio \& Valentino (2008), o parâmetro $\mathrm{m}$ do modelo está ligado à espessura adimensional da parte saturada pertencente à camada, variando de 0 a 1 . A espessura da camada saturada (m.H) está diretamente ligada à intensidade da precipitação. Liao et al. (2010) define a grade composta pelo talude infinito (ou seja, cada célula de trabalho) como um tanque de equilíbrio hídrico que leva em consideração, de maneira simultânea, tanto o ganho de água pela infiltração das chuvas, quanto a sua perda em virtude do escoamento através do elemento do talude. O cálculo do balanço hídrico em cada passo de tempo é estabelecido pela equação abaixo:

$$
\left\{\begin{array}{l}
\mathrm{m}_{1}=0 \\
\mathrm{O}_{\mathrm{t}}=K_{t} \cdot \sin \beta \cdot m_{t} \cdot H \cdot \cos \beta \cdot \Delta t \\
\Delta m_{t}=\frac{\left(I_{t}-O_{t}\right)}{n \cdot H \cdot\left(1-S_{r}\right)} \\
m_{t+1}=m_{t}+\Delta m_{t}
\end{array}\right.
$$

Nestas equações, $\mathrm{o}$ índice $\mathrm{t}$ refere-se à iteração atual para o balanço hídrico, $\Delta t$ é o intervalo de tempo, em segundos, e $\mathrm{m}_{1}$ corresponde ao valor inicial de $\mathrm{m}$, que pode ser nulo quando toda a camada de solo encontra-se em uma condição não saturada, como mostra a Equação 15, ou pode ter um valor não nulo, definindo a representação do nível freático inicial. $\mathrm{O}$ termo $\mathrm{m}_{\mathrm{t}}$ relaciona-se ao valor calculado em cada passo de tempo. $S_{\mathrm{r}}$ é o grau de saturação (em porcentagem), $\beta$ é a declividade do talude (em radianos) e $\mathrm{n}$ relaciona-se à porosidade do solo (em porcentagem). $\mathrm{O}_{\mathrm{t}}$ representa a saída de água de uma porção finita para um talude de comprimento finito L. A intensidade da chuva por unidade de tempo é dada por $\mathrm{I}_{\mathrm{t}}$, em $\mathrm{mm}$, sendo posteriormente transformada para metros, enquanto que capacidade global de drenagem, decorrente da permeabilidade intrínseca do solo e da existência de numerosos caminhos preferenciais de fluxo, corresponde ao termo $\mathrm{K}_{\mathrm{t}}$, dado em $1 / \mathrm{s}$.

\section{Modelo Tridimensional de Estabilidade de Taludes}

A técnica proposta utiliza os diversos layers provenientes de uma modelagem espacial com uso de uma plataforma pautada em sistemas de informação geográfica, sob o formato raster, para criar um modelo 3D baseado em colunas, por meio da discretização da massa em estudo. Nesta estrutura, para cada coluna de solo, os dados necessários para a execução da análise da estabilidade de taludes podem ser retirados a partir da posição da célula correspondente dentro das camadas rasterizadas.

Para a formulação do fator de segurança 3D, Qiu et al. (2006) utilizaram como base o modelo proposto por Xi et al. (2004), exposto na equação abaixo, o qual representa uma modificação do trabalho de Hovland (1977):

$$
F S 3 D=\frac{\sum_{J} \sum_{I}(c A+W \cos \theta \tan \phi) \cos \theta_{A v r}}{\sum_{J} \sum_{I}\left(W \sin \theta_{A v r} \cos \theta_{A v r}\right)}
$$


Na equação acima, FS3D refere-se ao fator de segurança tridimensional, $c$ é a coesão $\left(\mathrm{kN} / \mathrm{m}^{2}\right), A$ é a área da superfície de ruptura $\left(\mathrm{m}^{2}\right)$, W é o peso de uma coluna de solo $(\mathrm{kN})$, $\phi$ é o ângulo de atrito $\left({ }^{\circ}\right), \theta$ é a inclinação da superfície de ruptura $\left(^{\circ}\right)$,

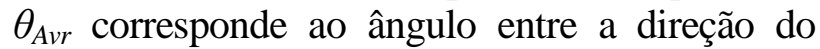
movimento e o plano horizontal $\left({ }^{\circ}\right)$, enquanto $J$ e $I$ retratam os números das linhas e colunas da célula dentro do intervalo da massa de ruptura do talude, respectivamente. Xi et al. (2004) definem quatro modelos para o cálculo do fator de segurança em relação às possíveis situações de ocorrência, cujo comportamento pode ser antecipado:

Modelo 1: a superfície de ruptura se forma na zona não-saturada, entre a frente úmida, cujo avanço se processa a partir da superfície do terreno, e o nível freático. Nessa situação, a força resistente horizontal $F_{1}$ e a força cisalhante horizontal $F_{2}$, ambas atuantes na superfície de ruptura, serão dadas pelas equações abaixo:

$$
\begin{aligned}
& F_{1}=\left\{c_{i}^{\prime} A^{\prime}+\left[\gamma_{i} z+\left(\gamma_{s a t}-\gamma_{i}\right) H_{w}\right] A \cos \theta \tan \phi^{\prime}\right\} \cos \theta_{A v r} \\
& F_{2}=\left[\gamma_{i} z+\left(\gamma_{s a t}-\gamma_{i}\right) H_{w}\right] A \sin \theta_{A v r} \cos \theta_{A v r}
\end{aligned}
$$

Modelo 2: a superfície de ruptura se forma na zona saturada definida entre a superfície do

terreno e a frente úmida. Para este caso, $\mathrm{F}_{1}$ e $\mathrm{F}_{2}$ são definidas pelas seguintes expressões:

$$
\begin{aligned}
& F_{1}=\left[c^{\prime}{ }_{w} A^{\prime}+\left(\gamma_{s a t} z \cos \theta-u_{w}\right) A \tan \phi^{\prime}\right] \cos \theta_{A v r} \\
& F_{2}=\gamma_{s a t} z A \sin \theta_{A v r} \cos \theta_{A v r}
\end{aligned}
$$

Modelo 3: a frente úmida atinge o nível freático, de forma que a superfície de ruptura se forma abaixo do nível do lençol. Assim, tem-se uma situação similar ao modelo 2, podendo-se utilizar as equações 19 e 20 para representar as forças $F_{1}$ e $F_{2}$, respectivamente.
Modelo 4: a formação da superfície de ruptura ocorre na parte saturada abaixo do nível freático, com a existência de uma zona não-saturada situada entre a frente úmida e o nível freático.

As equações a seguir mostram as forças $F_{1}$ e $F_{2}$ :

$$
\begin{aligned}
& F_{1}=\left\{c^{\prime}{ }_{w} A^{\prime}+\left[\left(\gamma_{i}\left(z_{w}-H_{w}\right)+\gamma_{s a t}\left(H_{w}+z-z_{w}\right)\right) \cos \theta-u_{w}\right] A \tan \phi^{\prime}\right\} \cos \theta_{A v r} \\
& F_{2}=\left[\gamma_{i}\left(z_{w}-H_{w}\right)+\gamma_{s a t}\left(H_{w}+z-z_{w}\right)\right] A \sin \theta_{A v r} \cos \theta_{A v r}
\end{aligned}
$$

Nas equações 17 a 22, $\gamma_{\text {sat }}$ corresponde ao peso específico do solo saturado $\left(\mathrm{kN} / \mathrm{m}^{3}\right), \gamma_{i}$ é peso específico do solo inicial $\left(\mathrm{kN} / \mathrm{m}^{3}\right), c^{\prime}{ }_{i}$ é coesão efetiva inicial do solo $\left(\mathrm{kN} / \mathrm{m}^{2}\right), c^{\prime}{ }_{w}$ refere-se à coesão efetiva saturada do solo $\left(\mathrm{kN} / \mathrm{m}^{2}\right), \phi$ ' é o ângulo de atrito efetivo do solo $\left({ }^{\circ}\right), z_{w}$ é profundidade do nível freático (m), $H_{w}$ é profundidade da frente úmida $(\mathrm{m}), \quad \mathrm{Z}$ relaciona-se a profundidade da superfície de ruptura $(\mathrm{m}), u_{w}$ é a poropressão $\left(\mathrm{kN} / \mathrm{m}^{2}\right), A$ é área da coluna de solo $\left(\mathrm{m}^{2}\right)$, A' é a área da superfície de ruptura da coluna de solo $\left(\mathrm{m}^{2}\right), \theta$ é a inclinação da superfície de ruptura $\left(^{\circ}\right)$ e $\theta_{A v r}$ corresponde ao ângulo de mergulho da direção principal de deslizamento $\left({ }^{\circ}\right)$.

O cálculo do fator de segurança 3D é realizado pelo somatório de $F_{1}$ e $F_{2}$ de todas as colunas de solo da massa em ruptura, considerando-se que não existe atrito lateral nas colunas de solo. Portanto, a expressão para o fator de segurança
3D é representada por:

$$
F S_{3 D}=\frac{\sum_{J} \sum_{I} F_{1}}{\sum_{J} \Sigma_{I} F_{2}}
$$

onde $F_{1}$ e $F_{2}$ são dadas em $\mathrm{kN}$. Para efeito de aplicação da Equação 23, as forças $F_{1}$ e $F_{2}$ de cada coluna são definidas mediante a verificação de qual situação se aplica, dentre as quatro citadas anteriormente, com o uso das equações mais apropriadas.

\section{Método Probabilístico Aplicado}

A formulação apresentada até então representa uma análise determinística, resultando, ao final, em um único valor de fator de segurança, responsável por mensurar a condição de estabilidade da encosta.

Propôs-se a incorporação do método probabilístico das Estimativas Pontuais (Rosenblueth, 1975) capaz de fornecer a distribuição probabilística para o fator de 
segurança 3D e, consequentemente, a medida da probabilidade de ruptura da encosta, que representaria um elemento adicional na análise da estabilidade.

O procedimento para a execução do método probabilístico considera as combinações das estimativas pontuais máximas $\left(\bar{X}_{l}+\sigma_{\mathrm{i}}\right)$ e mínimas $\left(\bar{X}_{l}-\sigma_{\mathrm{i}}\right)$ para cada variável independente $\mathrm{i}$, arbitrada como estatística, que compõe o cálculo do fator de segurança, onde se assume a média da variável em questão com sendo $\bar{X}_{l}$ e $\sigma_{i}$ refere-se ao seu desvio padrão, para certa célula da região.

Calculam-se os fatores de segurança para todas as combinações possíveis, o que permite a composição de uma amostra formada por $2^{\mathrm{i}}$ elementos, a partir da qual é possível estabelecer sua média e seu desvio padrão.

Assumindo-se uma distribuição normal de probabilidade para o fator de segurança, a probabilidade de ruptura pode ser obtida pelo cálculo da área da curva de probabilidade definida por valores de fatores de segurança 3D menores que o valor crítico unitário.

\section{Implementação Computacional}

De forma computacional, a implementação no MATLAB se processou principalmente em torno de quatro funções específicas (definição do nível freático inicial, identificação da localização de encostas individuais para análise, uso do modelo hidrológico e do modelo de estabilidade de taludes 3D probabilístico), todas coordenadas por uma rotina principal.

Inicialmente, o código principal é responsável por fazer a leitura e adequação dos dados de entrada necessários para a execução das análises. Esta adequação relaciona-se à organização dos dados espaciais em matrizes, bem como o ajuste das suas unidades.

Ressalta-se que os dados de chuva também devem ser apresentados sob o formato matricial, com as mesmas dimensões dos layers que representam a área de estudo, onde cada pixel terá um registro de chuva associado a uma dada porção do terreno.

Desta forma, ao simular um evento pluviométrico, são necessárias tantas grades quanto o número de passos de chuva envolvidos. Para a formulação do método probabilístico, ainda são requeridos valores de coeficiente de variação para a coesão efetiva, ângulo de atrito efetivo e peso específico do solo (variáveis consideradas com natureza estatística no desenvolvimento do método probabilístico), os quais são armazenados em um vetor específico.

Adicionalmente, uma matriz com a delimitação das encostas as quais deverão ser avaliadas também é necessária. As encostas que constarão do cálculo do fator de segurança deverão ser representadas por uma numeração sequencial, de modo que todos os pixels que fazem parte de uma determinada área a ser analisada serão representados por um mesmo valor.

Quando todos os dados de entrada se encontram carregados, a função responsável pela verificação da quantidade de encostas a serem analisadas e quais as células que fazem parte destas regiões é chamada.

A função retorna, como parâmetros de saída, as posições de todas as células válidas para cada encosta. Também, com a fase de input finalizada, utiliza-se a função responsável pela estimativa do nível freático inicial, o qual servirá de base para o modelo hidrológico de Green \& Ampt (1911).

Na sequência, aplica-se o modelo de infiltração a toda área de estudo com base nos dados de chuva impostos à simulação. Os comandos referentes ao modelo encontram-se organizados em uma função específica.

No caso em que a condição da Equação 14 não seja satisfeita, haverá a necessidade de empregar-se, como solução, um processo iterativo para o cálculo da quantidade acumulada de água infiltrada, o método de Newton-Rhapson. O número de iterações proposto foi estabelecido em 1000 com um erro aceitável inferior a 0,001.

A última função computacional desenvolvida diz respeito ao modelo de estabilidade de encostas tridimensional devidamente modificado para incorporar o método das estimativas pontuais.

Em termos da estrutura de resultados, duas abordagens foram admitidas: a primeira considerando o cálculo da estabilidade (e, consequentemente, a probabilidade de ruptura) apenas das encostas delimitadas a priori, e que constam como input, e a segunda, referente à aplicação do modelo e ao cálculo das PR, pixel a pixel, permitindo avaliar individualmente a situação de cada célula. 


\section{APRESENTAÇÃO DE DADOS}

A área de estudo analisada nesta pesquisa corresponde a uma extensão situada nas cidades de Sobradinho e Lago Norte, Distrito Federal, Brasil. Esta região é a mesma estudada por Azevedo et al. (2013), os quais processaram uma análise de susceptibilidade a ocorrência de deslizamentos.

Conforme estudos da defesa civil do Distrito Federal realizados em 2012, esta região apresenta pontos críticos de ocupação irregular, sendo classificados como áreas com grande potencial para ocorrência de escorregamentos.

Para a estruturação das informações espaciais da área citada, essenciais para a efetivação das análises, fez-se uso da plataforma computacional de sistemas de informação geográfica denominada Spring 5.3 (Câmara et al., 1996).

Neste software, foram criados, inicialmente, um banco de dados e um projeto, específicos para o presente trabalho.

As principais características relacionadas a este projeto encontram-se listadas a seguir:

Projeção e datum utilizados: UTM/SIRGAS 2000, zona 23 sul;

Retângulo envolvente em coordenadas planas:

$\mathrm{X} 1=189.490 \mathrm{~m} ; \mathrm{Y} 1=8.260 .775 \mathrm{~m}$;

$\mathrm{X} 2=204.000 \mathrm{~m} ; \mathrm{Y} 2=8.270 .559 \mathrm{~m}$;

A área de estudo encontra-se representada na Figura 2.


Figura 2 - Localização da área de estudo (Imagem de satélite oriunda do Google Earth).

Ressalta-se, ainda, que os planos de informação usados apresentaram pixels com resolução espacial de $5 \mathrm{~m}$. Desta forma, a área avaliada ficou representada por layers em formato matricial compostos por 1957 linhas e 2902 colunas.

Procedeu-se a importação dos dados do meio físico pertinentes ao estudo para o software Spring 5.3, organizando-os em categorias específicas e planos de informação distintos.

O conjunto utilizado de informações espaciais básicas concernentes à região analisada refere-se, primordialmente, à distribuição pedológica e à configuração do modelo numérico de terreno, do qual se pôde extrair a grade de declividades e orientação das vertentes.

A carta de solos incorporada a presente pesquisa tem como fonte $\mathrm{o}$ trabalho de atualização do mapa pedológico digital para o Distrito Federal empreendido pela Empresa Brasileira de Pesquisa Agropecuária (Reatto et al., 2004).

A quantificação dos parâmetros necessários para a aplicação do modelo, em relação a cada tipo de solo, foi realizada mediante a pesquisa de valores característicos destes elementos presentes na literatura técnica.

Obtiveram-se os valores de coesão efetiva e ângulo de atrito efetivo tomando-se como base o trabalho desenvolvido por Roque \& Souza 
(2008), no qual há uma compilação dos parâmetros citados para as unidades pedológicas existentes na área de estudo a partir da sua classificação e textura.

Tomando como referência a descrição dos solos expressa por Reatto et al. (2004), pôde-se, também, estimar a espessura de cada classe, enquanto dados de permeabilidade e peso espe- cífico foram obtidos de Castro (2011). Para frente úmida e teor de umidade volumétrico, considerou-se os valores propostos em Qiu et al. (2006).

A partir da carta de solos, observou-se a presença de sete tipos pedológicos constituindo a região de estudo conforme explicitado pela Figura 3.

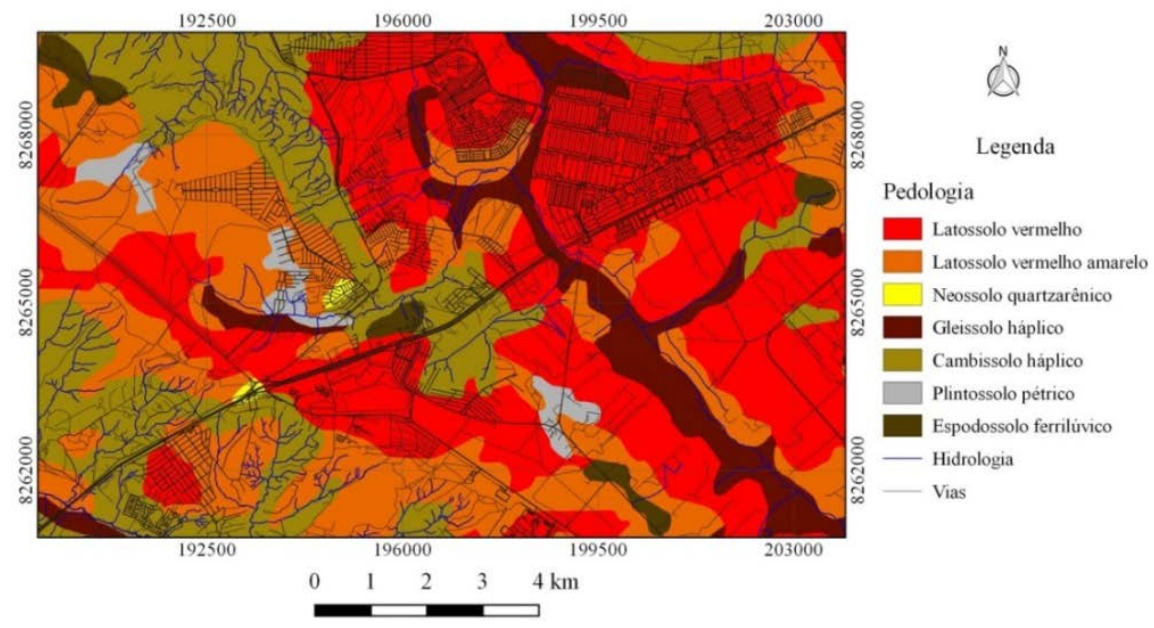

Figura 3 - Mapa de solos da região avaliada (Projeção / Datum: UTM zona 23 / SIRGAS 2000.

Valores de grau de saturação $\left(\mathrm{S}_{\mathrm{r}}\right)$ e (2011). As Tabelas 1-3 mostram os valores porosidade dos solos (n), requeridos pelo modelo, também foram obtidos de Castro adotados para os parâmetros envolvidos nas análises.

Tabela 1 - Características usadas para o cálculo do nível freático.

\begin{tabular}{l|c|c|c|c}
\hline \multirow{2}{*}{\multicolumn{1}{c|}{ Solo }} & \multicolumn{4}{|c}{ Parâmetros do solo } \\
\cline { 2 - 5 } & $\mathbf{K}_{\mathbf{s}}$ & $\mathbf{H}$ & $\mathbf{n}$ & $\mathbf{S}_{\mathbf{r}}$ \\
\cline { 2 - 5 } & $\mathbf{m} / \mathbf{s}$ & $\mathbf{m}$ & $\mathbf{\%}$ & $\mathbf{\%}$ \\
\hline Cambissolo háplico & $10^{-8}$ & 0,7 & 30 & 60 \\
\hline Espodossolo ferrilúvico & $10^{-9}$ & 4 & 40 & 95 \\
\hline Gleissolo háplico & $10^{-9}$ & 4 & 40 & 95 \\
\hline Latossolo vermelho & $10^{-6}$ & 3 & 60 & 40 \\
\hline Latossolo vermelho amarelo & $10^{-7}$ & 3 & 50 & 60 \\
\hline Neossolo quartzarênico & $2 \times 10^{-5}$ & 3 & 40 & 50 \\
\hline Plintossolo pétrico & $10^{-8}$ & 3 & 40 & 50 \\
\hline
\end{tabular}

Tabela 2 - Parâmetros de resistência dos solos e sucção mátrica na frente úmida.

\begin{tabular}{l|c|c|c|c}
\hline \multirow{2}{*}{\multicolumn{1}{c|}{ Solo }} & \multicolumn{4}{|c}{ Parâmetros do solo } \\
\cline { 2 - 5 } & $\mathbf{c}_{\mathbf{i}}^{\prime}$ & $\mathbf{c}_{\mathbf{w}}$ & $\boldsymbol{\phi} \boldsymbol{\phi}^{\prime}$ & $\boldsymbol{\Psi}_{\mathbf{f}}$ \\
\cline { 2 - 5 } & $\mathbf{k P a}$ & $\mathbf{k P a}$ & $\circ$ & $\mathbf{m}$ \\
\hline Cambissolo háplico & 5 & 4,5 & 25 & 0,5 \\
\hline Espodossolo ferrilúvico & 20 & 18 & 10 & 0,5 \\
\hline Gleissolo háplico & 20 & 18 & 10 & 0,5 \\
\hline Latossolo vermelho & 10 & 9 & 20 & 0,5 \\
\hline Latossolo vermelho amarelo & 10 & 9 & 20 & 0,5 \\
\hline Neossolo quartzarênico & 0 & 0 & 30 & 0,5 \\
\hline Pilntossolo pétrico & 20 & 18 & 10 & 0,5 \\
\hline
\end{tabular}


Tabela 3 - Valores de pesos específicos e teores de umidade volumétrico dos solos.

\begin{tabular}{l|c|c|c|c}
\hline \multirow{2}{*}{\multicolumn{1}{c|}{ Solo }} & \multicolumn{4}{|c}{ Parâmetros do solo } \\
\cline { 2 - 5 } & $\boldsymbol{\gamma}_{\mathbf{i}}$ & $\boldsymbol{\gamma}_{\text {sat }}$ & $\boldsymbol{\theta}_{\mathbf{i}}$ & $\boldsymbol{\theta}_{\mathbf{s}}$ \\
\cline { 2 - 5 } & $\mathbf{k P a}$ & $\mathbf{k P a}$ & - & - \\
\hline Cambissolo háplico & 15 & 19 & 0,4 & 0,68 \\
\hline Espodossolo ferrilúvico & 12 & 17 & 0,4 & 0,68 \\
\hline Gleissolo háplico & 12 & 17 & 0,4 & 0,68 \\
\hline Latossolo vermelho & 15 & 19 & 0,4 & 0,68 \\
\hline $\begin{array}{l}\text { Latossolo vermelho } \\
\text { amarelo }\end{array}$ & 15 & 19 & 0,4 & 0,68 \\
\hline Neossolo quartzarênico & 16 & 18 & 0,4 & 0,68 \\
\hline Plintossolo pétrico & 15 & 19 & 0,4 & 0,68 \\
\hline
\end{tabular}

Para o cálculo do desvio padrão, e, consequentemente, o estabelecimento das distribuições estatísticas das variáveis independentes da coesão, ângulo de atrito e peso específico do solo, empregadas no método das estimativas pontuais, fixou-se em $40 \%, 10 \%$ e $3 \%$ os valores de coeficientes de variação para estas variáveis, respectivamente, conforme Assis et al. (2012).

Para a geração do layer relativo ao nível freático, utilizou-se uma chuva uniforme de intensidade igual a $1 \mathrm{~mm} / \mathrm{h}$ com uma duração de 10 horas, no sentido de estabelecer uma condição inicial. Considerou-se que antes da aplicação deste evento pluviométrico a espessura adimensional da parte saturada da camada de solo seria nula $(\mathrm{m}=0)$.
A simulação subsequente, relativa à aplicação do modelo hidrológico para o cálculo da profundidade da frente úmida, correspondeu à aplicação de 3 horas de uma precipitação uniforme com intensidade de $20 \mathrm{~mm} / \mathrm{h}$. O layer resultante serviu de input para a análise de estabilidade, a qual foi realizada considerandose que a superfície de ruptura ocorre na profundidade máxima do solo.

Para a análise em relação às encostas individuais, selecionaram-se treze áreas, mostradas na Figura 4, baseando-se em pontos pertinentes do mapa de susceptibilidade proposto por Azevedo et al. (2013), correspondendo às regiões que alcançaram, no mínimo, $10 \%$ de probabilidade de ruptura na configuração de susceptibilidade.

\section{RESULTADOS E DISCUSSÕES}

Como resultados principais, destacam-se a quantificação e a distribuição da probabilidade de ruptura em relação às encostas delimitadas e ao estudo dos pixels isolados.

Em relação à análise das treze regiões estabelecidas, obtiveram-se valores de PR que variaram desde 16,3\%, correspondendo à encosta que se situa na porção sul da área de estudo, até medidas que ultrapassaram $50 \%$, como, por exemplo, os taludes localizados na porção oeste. Em comparação com os resultados de susceptibilidade expressos por Azevedo et al. (2013), em relação às mesmas áreas onde foram definidas as encostas, observa-se que houve uma tendência geral de crescimento da PR, fato comprovado pela execução da tabulação cruzada entre os respectivos layers das análises. Em 66,1\% das células há o aumento da PR, quando se faz a confrontação desde o cenário de susceptibilidade em direção à ameaça aqui elaborada, enquanto que, em outros $17,7 \%$, houve redução da PR.

A distribuição gerada pela análise de estabilidade de taludes 3D executada para cada pixel resultou em um panorama com um nível de ameaça, em termos gerais, superior às demais configurações encontradas. Com relação à distribuição espacial dos valores de PR alcançados, pode-se concluir que as previsões mais significativas apresentam uma ligação com relação à tendência de crescimento da declividade, de forma que a ameaça é visivelmente superior nas vertentes dos corpos d’água. Na Figura 5, encontra-se o mapa para a distribuição da PR para esta 


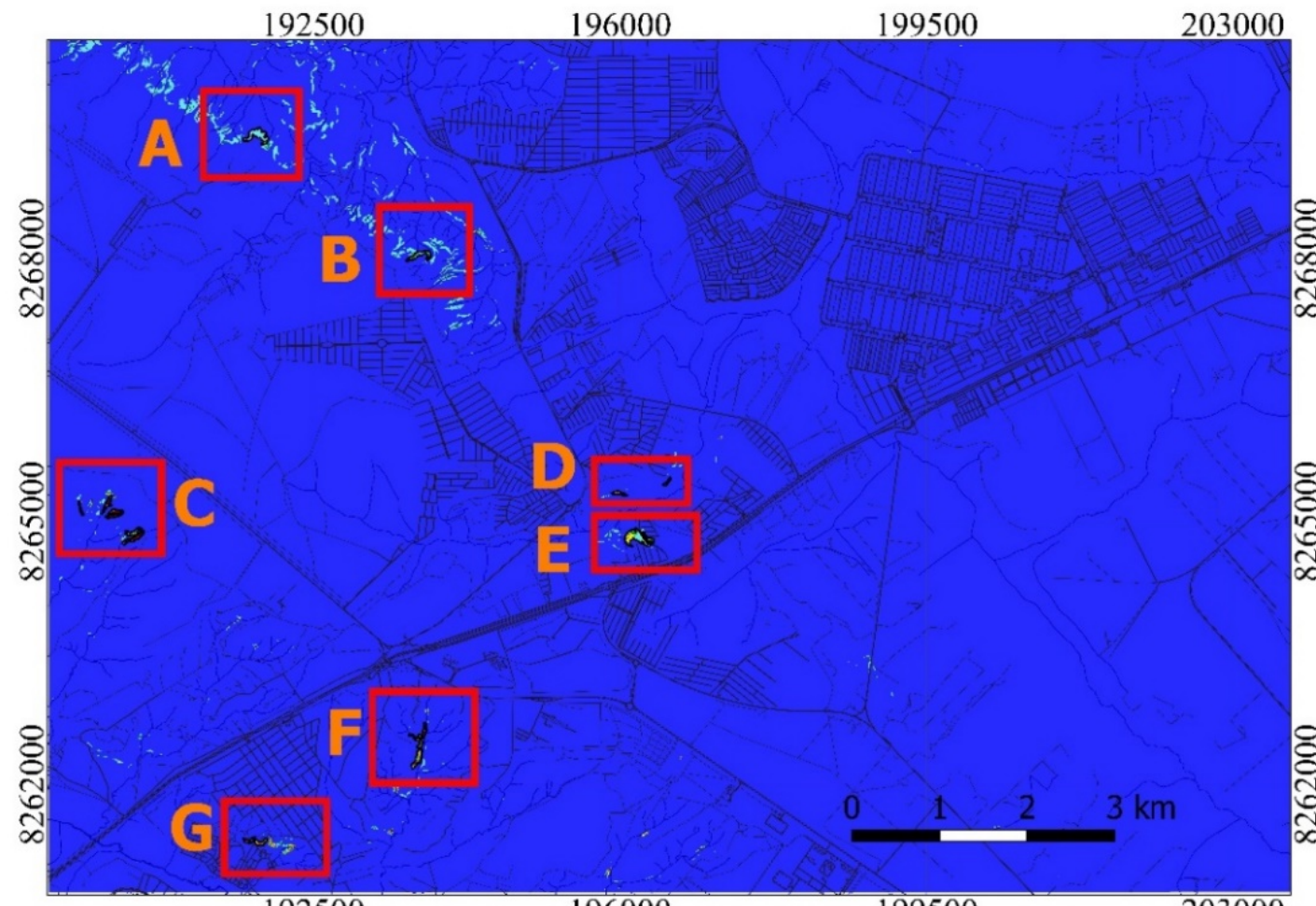

192500
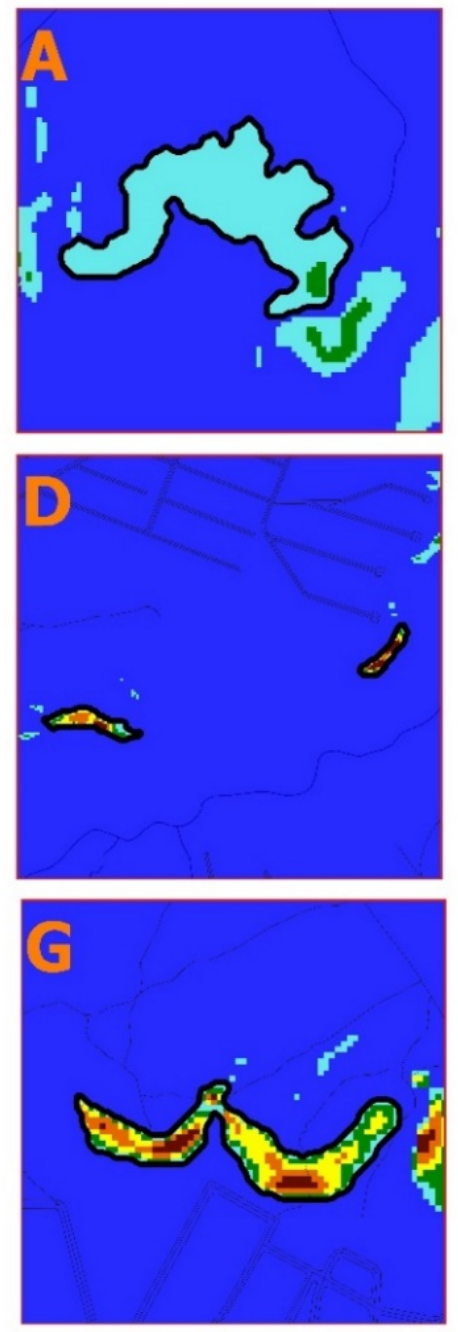

196000
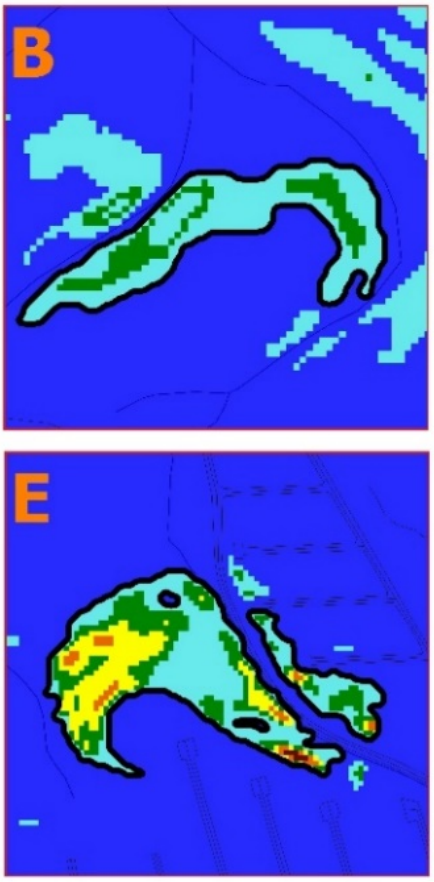

Legenda

\section{口Encostas}

Probabilidade de ruptura

- $0 \%$ a $10 \%$

$10 \%$ a $20 \%$

- $20 \%$ a $30 \%$

$\checkmark 30 \%$ a $40 \%$

- $40 \%$ a $50 \%$

- Maior que $50 \%$

- Hidrologia

Vias
199500

203000
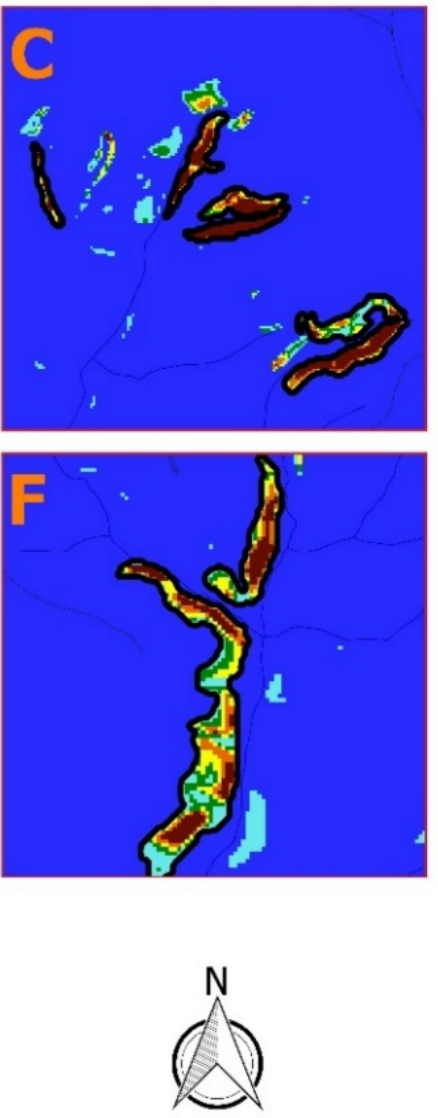

Projeção: UTM zona 23

Datum: SIRGAS 2000

Figura 4 - Localização das encostas analisadas em relação ao mapa de susceptibilidade proposto por Azevedo et al. (2013). 


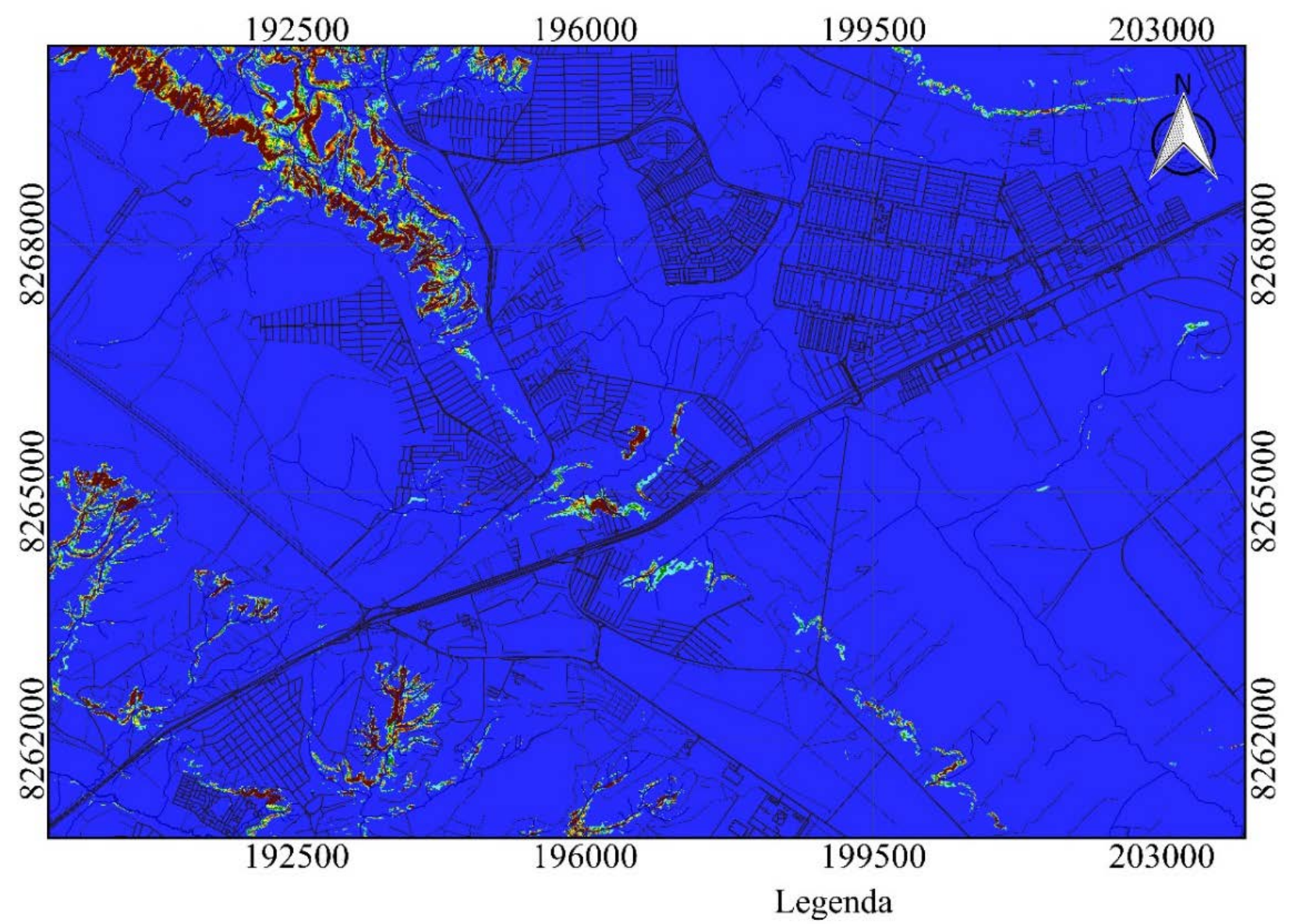

Projeção: UTM zona 23

Datum: SIRGAS 2000

$\begin{array}{lllll}0 & 1 & 2 & 3 & 4 \mathrm{~km}\end{array}$

Probabilidade de ruptura

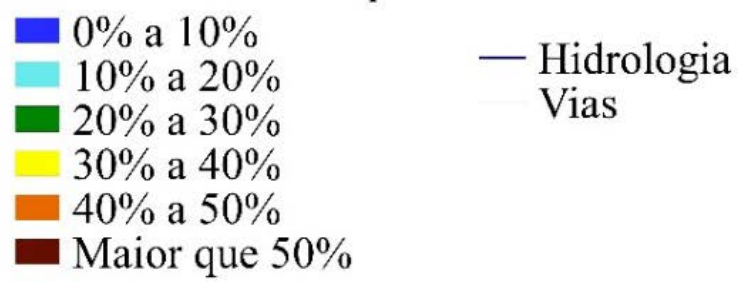

Figura 5 - Mapa com as probabilidades de ruptura geradas pela análise individual de cada pixel.

Tabela 4 - Classificação da área de estudo em função das probabilidades de ruptura obtidas.

\begin{tabular}{c|c|c}
\hline \multirow{2}{*}{$\begin{array}{c}\text { Categorias } \\
\text { de PR }\end{array}$} & \multicolumn{2}{|c}{ Área classificada } \\
\cline { 2 - 3 } & $\mathbf{k m}^{\mathbf{2}}$ & $\begin{array}{c}\text { Porcentagem da } \\
\text { área de estudo }\end{array}$ \\
\hline $\mathbf{0 \%}$ a 10\% & 135,2 & $95,2 \%$ \\
\hline $\mathbf{1 0 \%}$ a $\mathbf{2 0 \%}$ & 2 & $1,4 \%$ \\
\hline $\mathbf{2 0 \%}$ a $\mathbf{3 0 \%}$ & 1,2 & $0,8 \%$ \\
\hline $\mathbf{3 0 \%}$ a $\mathbf{4 0} \%$ & 0,8 & $0,6 \%$ \\
\hline $\mathbf{4 0 \%}$ a $\mathbf{5 0} \%$ & 0,7 & $0,5 \%$ \\
\hline$>\mathbf{5 0} \%$ & 2,1 & $1,5 \%$ \\
\hline
\end{tabular}

abordagem. Logo em seguida, a Tabela 4 traz a distribuição das medidas das categorias de PR geradas pela análise de estabilidade pixel a pixel.

Verifica-se que grande parte da região em estudo encontra-se classificada na categoria inferior de ameaça, que apresenta valores de PR variando de $0 \%$ a $10 \%$. A porcentagem de $4,8 \%$ restante encontra-se distribuída pelas classes de PR com valores mais altos.

Destaca-se a contribuição significativa dos valores de PR superiores a $50 \%$, os quais 
abrangem 1,5\% de toda região.

O que ocorre nestas células é a presença de fatores de segurança médios, relativos às distribuições de probabilidade, inferiores à unidade. Nestas condições, mais da metade da área da curva de probabilidade encontra-se à esquerda do fator de segurança crítico unitário para as encostas, o que caracteriza áreas com grande potencial para o desenvolvimento de instabilidades referentes a vertentes.

Fica evidente a ação da precipitação na definição do cenário de PR alcançado, quando se confronta a visões de susceptibilidade, conseguida por Azevedo et al. (2013), e de ameaça, obtida no presente trabalho. A incorporação do agente deflagrante na análise proporciona a mensuração da influência deletéria da chuva na estabilidade das encostas. Comparando-se a classificação da área de estudo entre os cenários citados, constata-se o crescimento, tratando-se da ameaça, de todas as classes que englobam valores superiores a $10 \%$ de PR, com redução da categoria correspondente de $0 \%$ a $10 \%$.

É possível observar também o surgimento de áreas com PR superiores a 10\% nas porções nordeste e leste da região analisada nesta análise de ameaça quando comparada com a carta de susceptibilidade.

O uso de um modelo hidrológico capaz de simular a ação da infiltração da chuva, com a consequente redução dos parâmetros de resistência do solo com a evolução do processo, mostra-se imprescindível para a representação realística do fenômeno estudado e para a geração de resultados que traduzam a condição de ameaça provocada por este agente detonante de movimentos de massa.

Verifica-se, ainda, que o resultado de estabilidade fornecido pela avaliação individual de pixels produz, em termos gerais, uma condição mais instável, quando comparada com os valores de PR obtidos para as mesmas regiões das encostas selecionadas.

Presume-se que a explicação se encontra na formulação utilizada no modelo: individualmente, para uma coluna de solo avaliada de forma isolada, a relação entre força favorável e contrária à estabilidade da encosta tende a uma condição limite de estabilidade.

De outra forma, ao se considerar a atuação conjunta das forças resistentes que atuam nas células que compõe o talude, em termos do seu somatório, isto determina uma condição mais estável frente à soma das forças desestabilizadoras, já que os pixels com melhores parâmetros de resistência do solo contribuem para sustentar aqueles onde a resistência não é tão satisfatória ou que possuem altos valores de forças atuantes.

Desta forma, deve-se considerar o tipo de avaliação a ser empregada em um dado estudo. Caso já exista um trabalho prévio, com a definição de áreas mais problemáticas ou mais susceptíveis a ocorrência de deslizamentos, pode-se aplicar a abordagem de delimitação dos taludes, ou, para se gerar o aspecto de distribuição referente à ameaça em toda área avaliada mediante a ação de um dado evento de chuva, utiliza-se o cálculo da estabilidade para cada pixel.

\section{CONCLUSÕES}

Com base nas análises neste trabalho, foi possível tecer algumas conclusões acerca do arranjo de ameaça que se configura pela região de estudo e da sua relação com alguns aspectos do meio físico, assim como sobre a integração estabelecida entre a utilização de uma plataforma de sistemas de informação geográfica e a ferramenta computacional responsável pela aplicação do método probabilístico das estimativas pontuais.

A abordagem de problemas referentes a estabilidades de taludes por meio da metodologia adotada mostrou-se prática e rápida, do ponto de vista computacional e da estruturação dos dados. O uso de sistemas de informação geográfica proporcionou a inserção de aspectos e características ambientais em um contexto espacial, possibilitando, ainda, uma manipulação mais facilitada destas informações.

O pós-processamento, com a elaboração das configurações resultantes, também foi uma das etapas onde esta plataforma forneceu grande auxílio.

O método probabilístico se destacou por permitir a inclusão da variabilidade de alguns parâmetros que compõem o modelo para a avaliação da estabilidade das encostas, fornecendo resultados de ameaça em termos de probabilidade de ruptura. A comparação entre os cenários estudados permitiu verificar a 
atuação da precipitação na estabilidade das encostas.

É notória a redução da condição de estabilidade dos taludes vinculados ao panorama de ameaça, quando comparado com o mapa de susceptibilidade apresentado por Azevedo et al. (2013). A chuva atua promovendo a redução dos parâmetros de resistência do solo e aumentando o peso do material referente à coluna avaliada, o que se traduz no acréscimo da PR.

Observa-se que áreas já definidas com valores significativos de susceptibilidade inicialmente, apresentaram um crescimento considerável de PR após a incorporação da precipitação, principalmente em regiões compostas por vertentes próximas às drenagens e declividades mais acentuadas.
Comparando-se as duas formas de avaliação (análise de encostas e de pixels individuais), verifica-se que a primeira fornece valores mais baixos de PR quando comparados com a estabilidade individual de cada célula.

A relação entre as forças resistentes e atuantes no talude apresenta-se a favor da segurança quando se admite a interação entre um conjunto de pixels, em nível de encostas.

Por fim, ressalta-se que o método seguido neste trabalho mostrou-se eficiente, sendo capaz de indicar as regiões com maior nível de ameaça para o desenvolvimento de eventos de deslizamentos de maneira quantitativa, podendo ser aproveitada como um instrumento voltado para o planejamento de pontos críticos, os quais se encontram sujeitos à ocupação e expansão urbana.

\section{AGRADECIMENTOS}

Os autores agradecem ao apoio financeiro concedido pela Comissão de Aperfeiçoamento de Pessoal do Nível Superior (CAPES), pelo Conselho Nacional de Desenvolvimento Científico e Tecnológico $(\mathrm{CNPq})$ e pela Fundação de Apoio à Pesquisa do Distrito Federal (FAP-DF) para realização deste trabalho.

\section{REFERÊNCIAS}

ASSIS, A.P.; ESPÓSITO, T.J.; GARDONI, M.G.; SILVA, P.D.E.A.; MAIA, J.A. (Coords). Métodos estatísticos aplicados a geotécnica. Brasília: Publicação G.AP-002/12 Programa de Pós-graduação em Geotécnica, 278 p., 2012.

AZEVEDO, G.F.; CARVAJAL, H.E.M.; SOUZA, N.M. Análise da Susceptibilidade à Ocorrência de Deslizamentos Rasos pela Utilização de Métodos Probabilísticos em Áreas de Risco do Distrito Federal. In: CONFERÊNCIA BRASILEIRA DE ENCOSTAS, 6, 2013, Angra dos Reis. Anais... Rio de Janeiro: Associação Brasileira de Mecânica dos Solos e Engenharia Geotécnica, 2013, p. 438-446.

CÂMARA, G.; SOUZA, R.C.M.; FREITAS, U.M.; GARRIDO, J.; MITSUO, F. Spring: integrating remote sensing and gis by object-oriented data modeling. Computers \& Graphics, v. 20, n.1, p. 395-403, 1996.

CASTRO, B.C. Modelo Geomecânico para os Principais Solos de Brasília. Brasília, 2011. 179 p. Dissertação (Mestrado em Geotécnica), Departamento de Engenharia Civil e Ambiental, Universidade de Brasília.

CHO, S.E. \& LEE, S.R. Evaluation of surficial stability for homogeneous slopes considering rainfall characteristics. Journal of Geotechnical and Geoenvironmental Engineering, v. 128, n. 9, p. 756-763, 2002.

COLLINS, B.D. \& ZNIDARCIC, D. Slope stability issues of rainfall induced landslides. In: MARIC, B.; LISAC, Z.; SZAVITS-NOSSAN, A. (Eds.), Geotechnical hazards: XI Danube-European conference on soil mechanics and geotechnical engineering. Rotterdam: Balkema, p. 791-798, 1998.

FRATTINI, P.; CROSTA, G.B.; FUSI, N.; NEGRO, P. Shallow landslides in pyroclastic soils: a distributed modeling approach for hazard assessment. Engineering Geology, v. 73, n. 1, p. 277-295, 2004.

GREEN, W.H. \& AMPT, G.A. Studies of soil physics, part I The flow of air and water through soils. The Journal of Agricultural Science, v. 4, n. 1, p. 1-24, 1911.
HOVLAND, H.J. Three-dimensional slope stability analysis method. Journal of the Geotechnical Engineering, v. 103, n. 9, p. 971-986, 1977.

LIAO, Z.; HONG, Y.; WANG, J.; FUKUOKA, H.; SASSA, K.; KARNAWATI, D.; FATHANI, F. Prototyping an experimental early warning system for rainfall-induced landslides in Indonesia using satellite remote sensing and geospatial datasets. Landslides, v. 7, n. 1, p. 317-324, 2010.

MONTRASIO, L. \& VALENTINO, R. A model for triggering mechanisms of shallow landslides. Natural Hazards and Earth System Sciences, v. 8, p. 1149-1159, 2008.

QIU, C.; ESAKI, T.; XIE, M.; MITANI, Y.; WANG, C. Spatiotemporal estimation of shallow landslide hazard triggered by rainfall using a three-dimensional model. Environmental Geology, v. 52, n. 8, p. 1569-1579, 2006.

REATTO, A.; MARTINS, E.S.; FARIAS, M.F.R.; SILVA, A.V.; CARVALHO JR., O.A.C. (Coordenadores). Mapa Pedológico Digital - SIG Atualizado do Distrito Federal Escala 1:100.000 e uma Síntese do Texto Explicativo. Planaltina: Embrapa, 29 p., 2004.

ROQUE, W.V. \& SOUZA, N.M. Análise da Estabilidade de Taludes pelo Método Racional na Região do Taquari (DF). In: CONGRESSO BRASILEIRO DE GEOLOGIA DE ENGENHARIA E AMBIENTAL, 12, 2008, Porto de Galinhas. Anais... Porto de Galinhas: ABGE, 2008, p. 1-9.

ROSENBLUETH, E. Point estimates for probability moments. Proceedings of the National Academy of Sciences of the United of America, v. 72, n. 1, p. 3812-3814, 1975.

SCHUSTER, R.L. Socioeconomic significance of landslides. In: TURNER, A.K. \& SCHUSTER, R.L. (Eds.), Landslides: Investigation and Mitigation. Washington: National Academy Press, p. 12-35, 1996.

SERRANO, S.E. Improved decomposition solution to Green and Ampt equation. Journal of Hydrologic Engineering, v. 8, n. 3, p. 158-160, 2003. 
TOMinAGA, L.K. Avaliação de Metodologias de Análise de Risco a Escorregamentos: Aplicação de um Ensaio em Ubatuba, SP. São Paulo, 2007. 220p. Tese (Doutorado em Geografia Física) - Faculdade de Filosofia, Letras e Ciências Humanas, Universidade de São Paulo.

XIE, M.; ESAKI, T.; CAI, M. A GIS-based method for locating the critical 3D slip surface in a slope. Computers and Geotechnics, v. 31, n. 1, p. 267-277, 2004.
XIE, M.; LIU, X.; ESAKI, T.; WANG, Z.; WANG, J. Threedimensional critical slip surface identification and slope stability assessment of 11\# lava lobe of unzen volcano, Japan. Italian Journal of Engineering Geology and Environment, v. 3, n. 1, p. 1023-1030, 2011.

Submetido em 16 de setembro de 2016 Aceito em 8 de março de 2018 